Dieses Dokument ist eine Zweitveröffentlichung (Postprint) /

This is a self-archiving document (accepted version):

Thomas Stoppe, Matthias Bornitz, Nikoloz Lasurashvili, Kirsten Sauer, Thomas Zahnert, Karim Zaoui, Thomas Beleites

Function, Applicability, and Properties of a Novel Flexible Total Ossicular Replacement Prosthesis With a Silicone Coated Ball and Socket Joint

Erstveröffentlichung in / First published in:

Otology \& Neurotology. 2018, 39(6), S. 739-747. ISSN 1537-4505.

DOI: https://doi.org/10.1097/MA0.0000000000001797

Diese Version ist verfügbar / This version is available on:

https://nbn-resolving.org/urn:nbn:de:bsz:14-qucosa2-726731 


\title{
Function, Applicability, and Properties of a Novel Flexible Total Ossicular Replacement Prosthesis With a Silicone Coated Ball and Socket Joint
}

\author{
Thomas Stoppe ${ }^{1}$, Matthias Bornitz ${ }^{1}$, Nikoloz Lasurashvili ${ }^{1}$, Kirsten Sauer ${ }^{1}$, Thomas Zahnert ${ }^{1}$, Karim Zaoui ${ }^{2}$, and \\ Thomas Beleites ${ }^{1}$ \\ ${ }^{1}$ Technische Universität Dresden, Carl Gustav Carus Faculty of Medicine, Otorhinolaryngology, Dresden \\ ${ }^{2}$ Heidelberg University Hospital, Otorhinolaryngology, Head and Neck Surgery, Heidelberg, Germany
}

\section{Introduction}

Passive prostheses in middle ear surgery have great variety and can be used for the reconstruction of different parts of the ossicular chain. Total ossicular replacement prostheses (TORP) and partial ossicular replacement prostheses (PORPs) involve alloplastic replacements for destroyed ossicles (1; $2 ; 3)$. Presently, they are commonly made of titanium 5 or other biocompatible materials, for example, ceramic glass (see, e.g., $(4 ; 5)$ ).

The length of the prosthesis must match the gap length in the ossicular chain; otherwise, it will cause pretensioning of the stapes annular ligament during implantation (6). High pretension (too long TORP) reduces the pressure buffering capability of the tympanic membrane (TM) due to worse mobility (7). Tension changes, e.g., because of scars or static pressure variations (postoperative aeration problems; (8)), can lead to a dislocation of the TORP from the tympanic membrane and/or the stapes footplate, damage to the stapes annular ligament or stapes footplate, and may cause conductive hearing loss (9; $10 ; 11)$.

Other medical factors, such as complications or ventilation problems, regardless of the prosthesis length and design, can also affect hearing results after surgery $(12 ; 13 ; 14)$.

In contrast to the common rigid TORP, the ossicular chain has two joints-the incudomallear joint (IMJ) and the incudostapedial joint (ISJ) - that allow a defined movement and sound transfer within the ossicular chain. This movement reduces the load to the stapes annular ligament during static pressure changes (15). As a result, less stiffening of the stapes annular ligament and less degradation of sound transfer through the ME under static pressure load can be achieved. The aim of prosthesis development is to achieve a middle ear reconstruction with similar behavior as the intact ossicular chain, especially at big varying static pressure of more than $2 \mathrm{kPa}$. In this case, the quasi-static movement of the tympanic membrane changes the distance between the stapes footplate and the malleus handle, resulting in an increase or decrease of tension at the prosthesis coupling points (stapes footplate and malleus handle). The development of a TORP that is capable of mimicking the function of the ossicular joints is consequential.

Recently, novel mechanisms related to middle ear prosthesis have been investigated, e.g., for the easy adjustment of the proper length of the prosthesis during implantation $(16 ; 17 ; 18)$ and for securing sound transmission and preventing damage to the stapes footplate and the ligament at ambient pressure loads after implantation $(19 ; 20 ; 21)$.

Additionally, the ease of insertion could benefit from such improved designs, which are assumed to be more important than the kind of material or a specific weight (22). New prostheses should address important influencing factors such as adaption to inter-individual anatomical variability and proper coupling (12). In terms of better coupling, a malleus notch is said to be beneficial $(23 ; 13)$.

Upcoming prosthesis designs should focus on a better long-term stability of the reconstruction. Previous investigations have shown that a ball joint is able to reduce the risk of damage at large movements of the TM $(24 ; 11 ; 21)$. The difference between the flexible design shown in this study and the other ball joint types developed recently (e.g. (21)) is the additional use of silicone covering for the ball and socket joint which is positioned in the middle of the prosthesis shaft. This construction introduces a reset movement, compared to the other designs, meaning that the deflected prosthesis head without external load again rises up to its initial position. This work investigates whether this flexible TORP design is a step towards mimicking the healthy ossicular chain. 


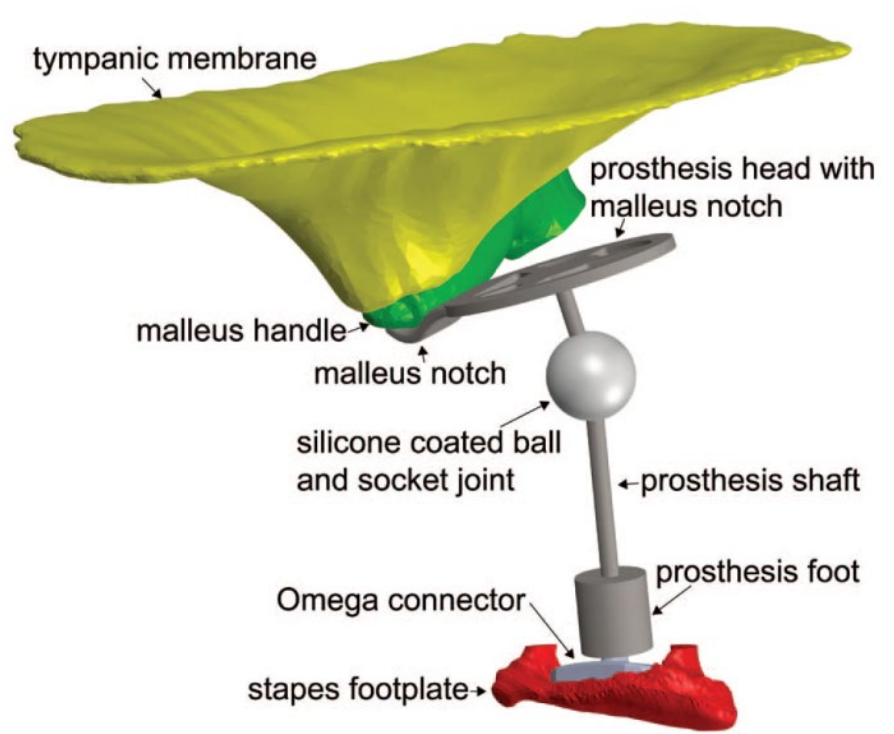

Figure 1: The flexible total ossicular replacement prosthesis (TORP) design and its placement in the ossicular chain in a 3D computer-aided-design-model; the notch of the TORP is attached at the malleus (green) and the prosthesis foot is placed on the Omega Connector on the stapes footplate (red).

\section{Materials and methods}

\subsection{Prosthesis design}

The flexible TORP construction (unapproved and still an investigational product) is related to the columella and extracolumella of birds, e.g. the ostrich (20). It is based on a standard rigid TORP design (the malleus notch TORP from the Heinz Kurz GmbH Medizintechnik, Dußlingen, Germany). A ball and socket joint is added inside the prosthesis shaft. This joint consists of a titanium ball that is connected to a titanium ball socket, enclosing about half of the ball (Fig. 1). It is coated with silicone for implementing a viscoelastic component that provides a reset force and joint stability. The ball and socket joint offers the necessary rigidity for sound transfer, while it slides at larger forces, e.g. at static pressure changes, to compensate for the tympanic membrane movement at pressure changes. A solely flexible prosthesis shaft (e.g. like a spring) won't provide adequate sound transmission $(25 ; 26)$.

The stiffness of the prostheses (measured between notch and foot) is angle dependent and determined with relaxation measurements to be at a maximum of about $38 \mathrm{~N} / \mathrm{m}$ (27). These values are lower than the one-axial stiffness of the IMJ (lateral-medial, about $100 \mathrm{~N} / \mathrm{m}$ to $1500 \mathrm{~N} / \mathrm{m}$; (28)) and the stapedial annular ligament stiffness (between $120 \mathrm{~N} / \mathrm{m}$ and about $4000 \mathrm{~N} / \mathrm{m},(29 ; 30 ; 6)$ ). Therefore, the constructive parameters (e.g. length, stiffness) are beneficial for low tensions and lower the risk of damage to the stapes annular ligament or the footplate. The sound transfer characteristics will be obtained in this study.

The investigated prostheses had a full length of $4.5 \mathrm{~mm}, 4.75 \mathrm{~mm}$ and $5 \mathrm{~mm}$, depending on the temporal bone morphology. This prosthesis concept aims to use a flexible element in combination with special attachment/fixation at the stapes footplate and the $\mathrm{TM} /$ malleus handle. At the stapes footplate, an osseointegrated coupling element, as proposed by (31), is a preferred solution. In this study, an omega connector (Kurz Medizintechnik, Dußlingen, Germany) was used. For better coupling at the TM, a prosthesis plate with a notch for the malleus was used. Such a modular prosthesis concept adds an additional joint-like attachment to the reconstruction, allowing for more degrees of freedom and a better connection to the stapes footplate. The malleus notch on the head plate enables an easy connection to the malleus handle (23).

\subsection{Preparation of temporal bones}

Nine fresh adult human temporal bones were used. The average age was about 42 (27-60) years for all nine and 45 (30-60) years for five reconstructed temporal bones. The specimens were prepared within 10 days post mortem. Mastoidectomy and posterior tympanotomy were performed for each temporal bone. For a better view of the stapes footplate, the facial nerve was removed. A reflective foil with a 
size of about $0.5 \mathrm{~mm} 2$ was placed at the center of the stapes footplate for measurements with a laser Doppler vibrometer (LDV). The ossicles, ligaments and tendons of the ossicular chain were kept intact. A hole was drilled into the anterior side of the ear canal to place a probe microphone in front of the TM. In the course of the measurements, parts of the inner ear were removed to gain access to the footplate from this side. Starting from the inner ear canal, the bone of the vestibulum labyrinthi was removed with a drill and the footplate was exposed. After taking the measurements for the intact middle ear, the ossicular chain was partly removed for a type III tympanoplasty. The incudostapedial joint was cut open and the incus was carefully removed with a hook and forceps. The stapes suprastructure was cut on both crura with a CO2-Laser (Illumina 755 Heraeus Lasersonics, Milpitas, CA, USA) so that only the footplate remained. Neither the annular ligament nor the stapes footplate were damaged or luxated during the preparation. This outcome represents one potential finding for a type III tympanoplasty. Other situations with removed malleus head, completely missing malleus or TM reconstruction were not considered. The ossicular chain reconstruction for each temporal bone was performed with a rigid TORP and a flexible TORP. The omega connector was used for a stable connection of the prosthesis at the stapes footplate $(32 ; 33)$. The notch at the prosthesis head was coupled onto the area between umbo and the middle of the manubrium, depending on the anatomical situation.

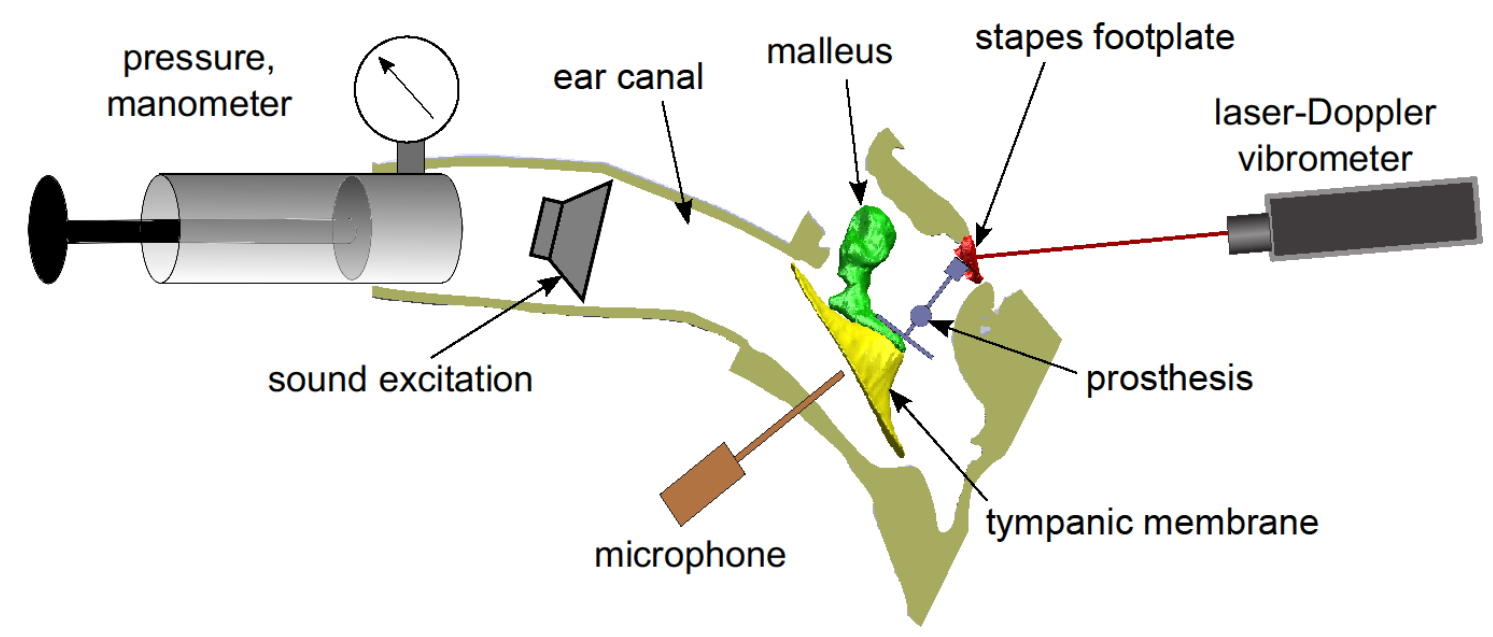

Figure 2: Measurement setup for ascertaining the middle ear transfer function (METF) from the side of the inner ear with pressure application and sound excitation in the ear canal.

\subsection{Experimental setup}

The experimental setup, shown in Fig. 2, was similar to the standard setup for middle ear transfer function (METF) measurements (34), except that additionally static pressure could be applied. An insert earphone connected to the ear canal delivered the sound signal. The applied sound pressure was measured with a probe microphone (ER-7C, Etymotic Research, Elk Grove Village, II) placed within an approximate distance of $3 \mathrm{~mm}$ to the TM.

The stapes footplate velocity was measured with LDV (CLV 700, CLV 1000 with modules M300, M050 and M003, Polytec, Germany) and then mathematically integrated to get the displacement of the stapes footplate. Static pressure could be applied to the ear canal with a pressure pump. Positive pressure in the ear canal is equivalent to a negative pressure in the tympanic cavity and vice versa. This simulates pressure changes, which may occur in healthy and pathological ears, e.g. while blowing the nose, using an elevator or a permanent negative pressure in the tympanic cavity in a Eustachian tube dysfunction $(35 ; 36 ; 7)$. Either positive or negative pressure of up to $2.5 \mathrm{kPa}$ was applied in the ear canal. This was over half of the maximum pressure during tympanometry $(4 \mathrm{kPa})$. It was the maximum 
pressure that could be applied without leakage for all the specimens, although all connections to the ear canal (earphone and microphone) were tightly sealed.

For measuring METFs, a multi-sinus excitation signal was applied with the insert earphone at about 90 dB SPL between $200 \mathrm{~Hz}$ and $5 \mathrm{kHz}$. A standard METF, displacement of the stapes footplate with reference to the applied sound pressure, was obtained for the intact and the reconstructed middle ears. METFs were measured with and without additionally applied static pressure.

For data evaluation, the METFs were mostly displayed in respect to a reference METF in a dB scale: $20^{*} \lg$ (METF/reference_METF). The references were the METFs of the intact middle ear, of the initial reconstruction or the previous METF without applied static pressure. These results were also reduced to mean values over audiometry frequencies $(0.5 ; 1 ; 2 ; 3 \mathrm{kHz})$, the pure tone average (PTA) according to American Academy of Otolaryngology-Head and Neck Surgery guidelines (37).

\subsection{Experimental protocol}

The sequence of measurements was as follows:

1. Measurement of METF of the intact middle ear, with LDV from the side of the tympanic cavity (TC).

2. Removal of the inner ear. METF of the intact middle ear measured at $0 \mathrm{kPa}$ (normal pressure). LDV measurement from the side of the inner ear.

3. A sequence of three static pressure application cycles from $+2.5 \mathrm{kPa}$ to $-2.5 \mathrm{kPa}$ was performed to simulate common ambient pressure changes.

4. METF measurement at $0 \mathrm{kPa}$ (normal pressure) was repeated. METFs before and after the pressure cycles were the same, indicating that these pressures did not damage the normal middle ear.

5. METF measured from the side of the inner ear with an applied static pressure of $+2.5 \mathrm{kPa}$ and $-2.5 \mathrm{kPa}$, respectively.

6. METF measurement at $0 \mathrm{kPa}$ (normal pressure) was repeated.

7. Incus and stapes suprastructure were removed and a standard TORP was inserted.

8. METF measured at normal pressure.

9. A sequence of three static pressure application cycles from $+2.5 \mathrm{kPa}$ to $-2.5 \mathrm{kPa}$ was performed to simulate common ambient pressure changes.

10. METF measurement at $0 \mathrm{kPa}$ (normal pressure) was repeated to check whether the pressure changes had altered the reconstruction (prosthesis position and coupling).

11. METF measurements from the side of the inner ear with static pressure of $+2.5 \mathrm{kPa}$ and -2.5 $\mathrm{KPa}$, respectively.

12. METF measurement at $0 \mathrm{kPa}$ (normal pressure) was repeated.

13. The TORP with flexible joint was inserted and the measurements from points 8 to 12 were repeated.

The temporal bones were kept moist during the experiments. Four specimens got damaged during the course of the measurements. These were excluded from the analysis. Five of the nine temporal bones were used for a comparison between the flexible TORP and the standard TORP reconstruction.

\section{Results}

The measured METFs of the intact ears (Fig. 3) matched the normal range of other studies (34). Therefore, the specimens were all regarded as non-pathological. In removing the inner ear, the magnitude of the METF increased (mainly at higher frequencies) and the first middle ear resonance (around $800 \mathrm{~Hz}$ ) became more prominent.

The METFs of the reconstructed middle ears with the flexible TORP were very close to the METFs of the intact ears (Fig. 4A). The corresponding PTA was $2.8 \mathrm{~dB}$ below the intact one (Table 1). There was 
also a little inter-individual variation. The reconstructions with the rigid TORP were somewhat worse. The PTA was $4 \mathrm{~dB}$ below the intact ear (Table 1 ) and there was considerably more individual variation as individual METFs were up to $20 \mathrm{~dB}$ below the intact ear.

After three pressure load cycles, the METFs for the flexible TORPs showed nearly no difference compared to the METFs measured directly after the implantation (Fig. 5). The PTA reduced by about $1.1 \mathrm{~dB}$ with small standard deviation. The PTA for the rigid TORPs decreased by about $1.8 \mathrm{~dB}$ but showed considerably more variation. The METFs value of individual reconstructions reduced by up to about 10 $\mathrm{dB}$. It can be assumed that the flexible TORP better maintains its position and coupling conditions.
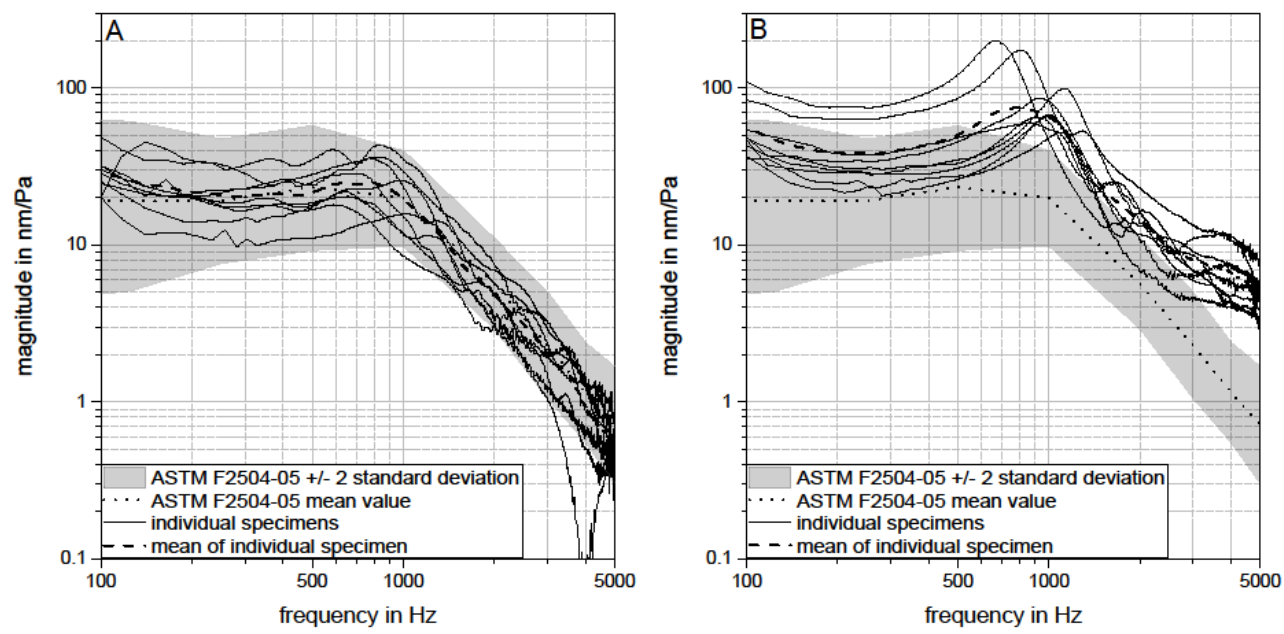

Figure 3: Middle ear transfer functions (METFs) of the intact temporal bones with inner ear (A) and measured from the inner ear side without the cochlea (B), in comparison to METF of other studies (Rosowski et al., (34)).
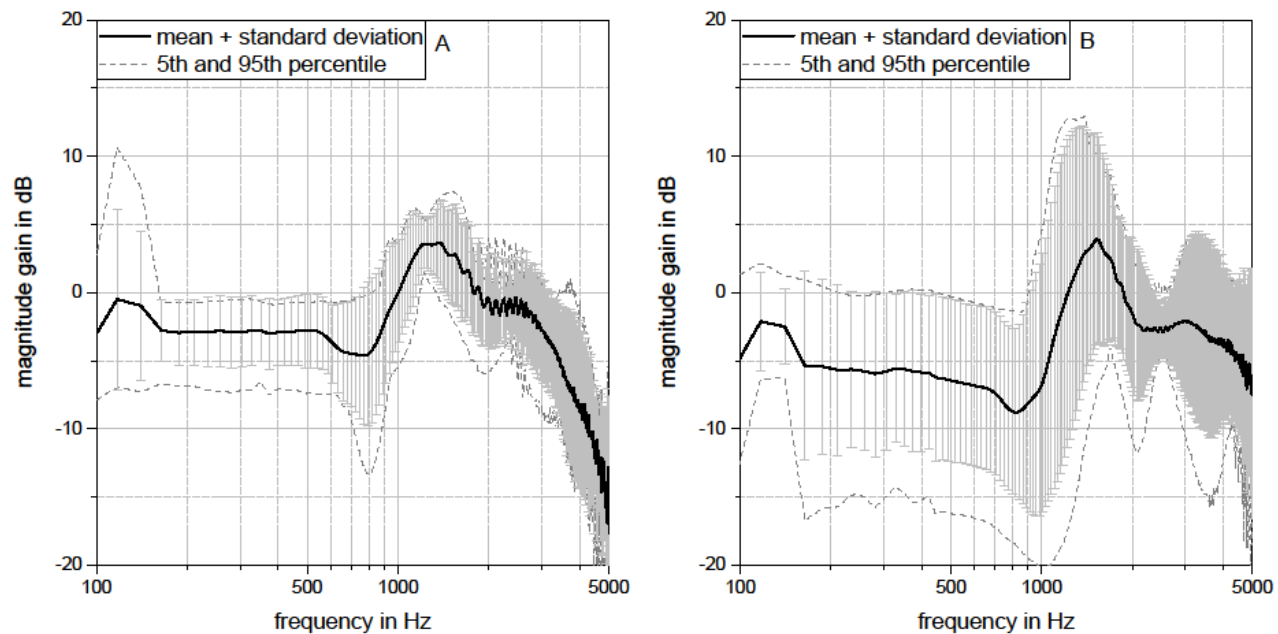

Figure 4: Middle ear transfer function (METF) magnitude gain of flexible total ossicular replacement prostheses (TORPs) (A) and rigid TORPs (B) at normal pressure directly after implantation. The $0 \mathrm{~dB}$ line represents the intact temporal bone middle ear transfer function (METF) with removed cochlea without applied pressure. The mean, standard deviation and the 5th and 95th percentiles of the reconstructed temporal bones are shown.

In the case of a permanent static pressure of $+/-2.5 \mathrm{kPa}$ in the intact middle ear, the METF magnitude at lower frequencies (below $1500 \mathrm{~Hz}$ ) reduced on an average by $15 \mathrm{~dB}$ to $20 \mathrm{~dB}$ (Fig. 6). The PTAs were $-9.8 \mathrm{~dB}$ and $-9.4 \mathrm{~dB}$. Over $2 \mathrm{kHz}$, the METFs did not show relevant changes compared to the METFs at normal pressure. This applied for the positive as well as the negative pressure and was related to the increased stiffness of the tympanic membrane and the stapes annular ligament.

Fig. 7 shows the changes in METFs at the negative and positive pressures of $2.5 \mathrm{kPa}$ in the ear canal after tympanoplasty type III with the rigid and the flexible TORPs. 
A positive pressure of $2.5 \mathrm{kPa}$ in the ear canal (equivalent to the negative pressure in the TC, Fig. 7, top figures) caused a characteristic decrease in the METF at frequencies lower than $1.5 \mathrm{kHz}$. With the flexible TORP, the changes in the METF were about the same as for the intact middle ears. The PTA was 7.9 $\mathrm{dB}$ below the intact middle ear. For the rigid TORP, the PTA reduction of $-19.3 \mathrm{~dB}$ was larger and the variation across specimens was also bigger.

At a pressure level of $-2.5 \mathrm{kPa}$ in the ear canal (equivalent to the positive pressure in the TC, Fig. 7, bottom figures), some prostheses lost their contact with the stapes footplate or the TM. This caused an individual loss of more than $35 \mathrm{~dB}$ in the METF magnitude and occurred with both TORP types. Accordingly, the PTA for the flexible TORP was $-18.7 \mathrm{~dB}$ and $12.4 \mathrm{~dB}$ for the rigid TORP. The reduction for the rigid TORP reconstruction was about $6 \mathrm{~dB}$ less than that for the flexible TORP, which showed greater variability.
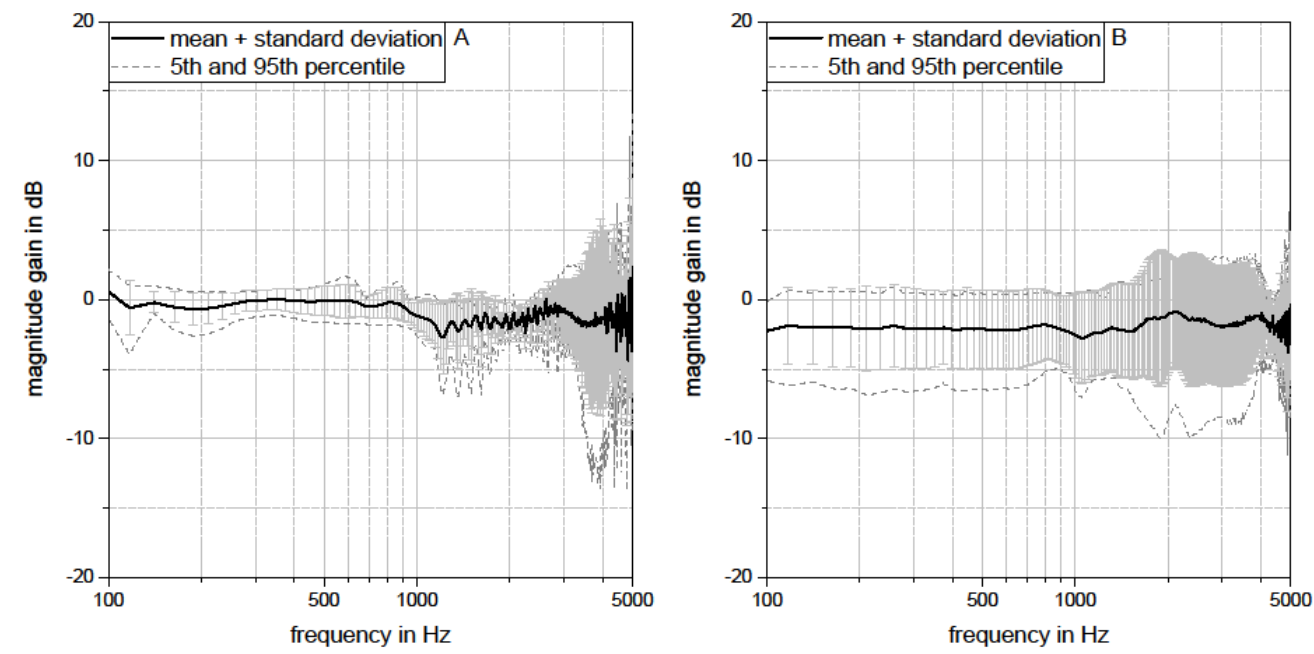

Figure 5: Middle ear transfer function (METF) magnitude gain in $\mathrm{dB}$ after three positive and negative pressure cycles for flexible total ossicular replacement prostheses (TORPs) (A) and rigid TORPs (B) at normal pressure. The $0 \mathrm{~dB}$ line represents each reconstructed TORP METF right after implantation. The mean, standard deviation and the 5th and 95th percentiles of the measurements are shown.
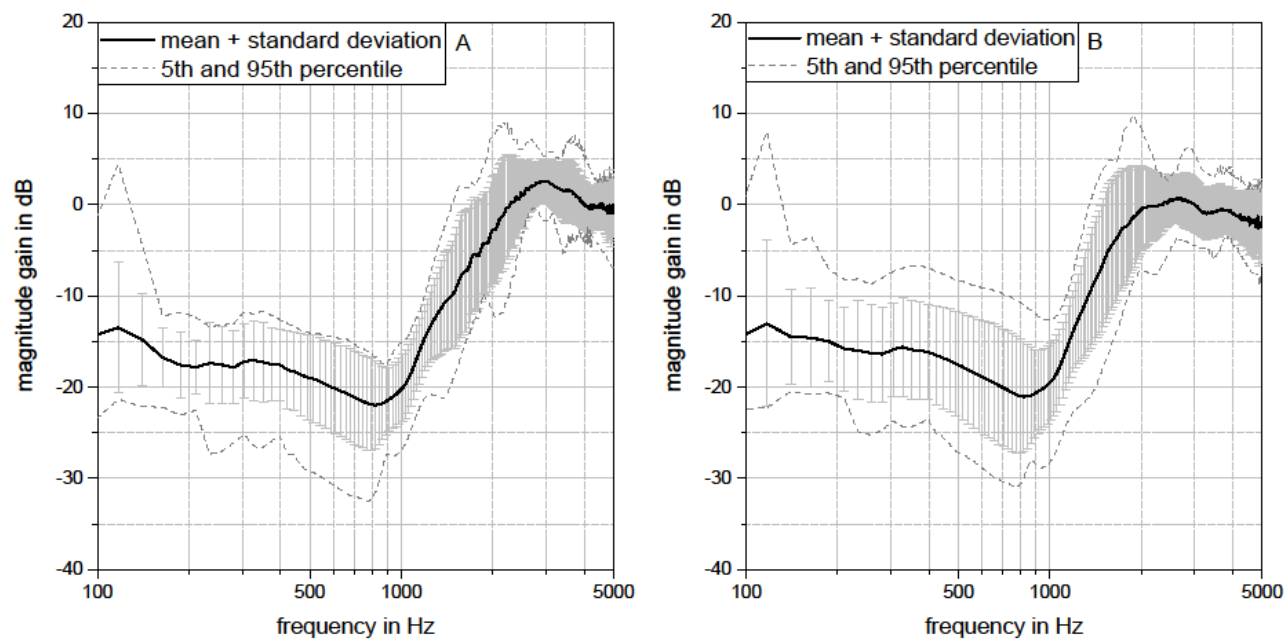

Figure 6: The middle ear transfer function (METF) magnitude gains of the nine intact temporal bones (without inner ear) at positive (A) and negative (B) pressure of about $+/-2.5 \mathrm{kPa}$ in the tympanic cavity (TC). The $0 \mathrm{~dB}$ line represents the METF of the intact temporal bones without applied pressure. The mean, standard deviation and the 5th and 95th percentiles are shown. 

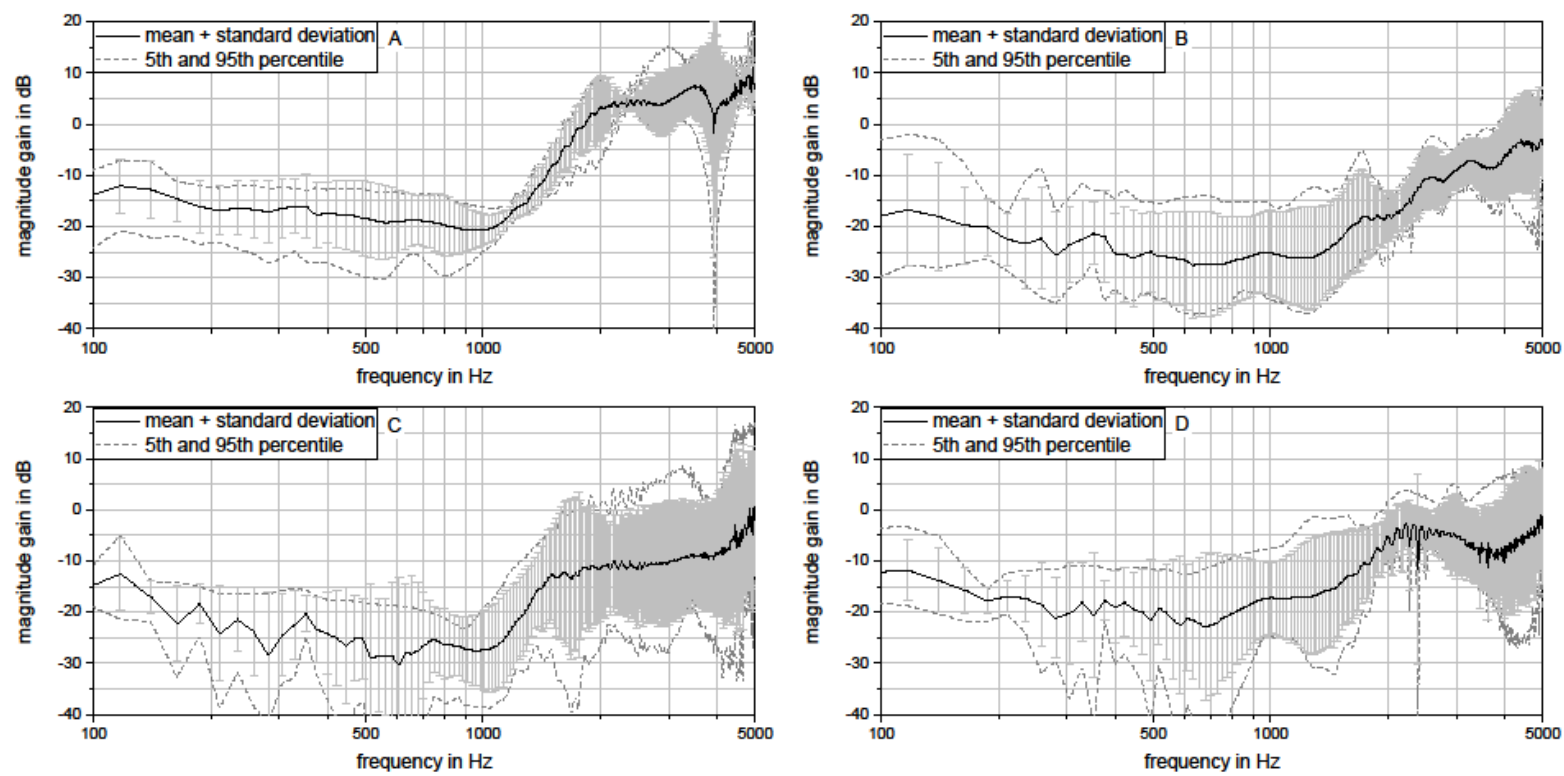

Figure 7: The middle ear transfer function (METF) magnitude gains of the five flexible total ossicular replacement prostheses (TORPs) (left figures) and five rigid TORPs (right figures) at positive (bottom figures) and negative (top figures) pressure of about +/- $2.5 \mathrm{kPa}$ in the tympanic cavity (TC). The $0 \mathrm{~dB}$ line represents the METF of the reconstructed temporal bones without cochlea without applied pressure after three load cycles. The mean, standard deviation and the 5th and 95th percentiles are shown.

\section{Discussion}

\subsection{Reconstruction and METF of the reconstructed middle ear}

This experimental study was confined to type III tympanoplasties of middle ears with intact tympanic membrane and complete malleus. For this anatomical condition, the expected advantages of the flexible TORP pertain to easier placement and a reconstruction that requires less pretension of the stapes annular ligament. In another situation, in which the malleus or a part of it is missing and the TM has lost its pre-stressed shape, there may be no initial benefit with a flexible TORP.

In order to fit the prosthesis with pretension and to obtain a stable reconstruction, normal rigid TORPs that are slightly longer than necessary are chosen usually. Earlier experiments have indicated a reduction of the METF at frequencies below $1.5 \mathrm{kHz}$ (38). Recently, Neudert et al. (6) have shown that an increase in the prosthesis length by $200 \mu \mathrm{m}$ results in about $25 \mathrm{~dB}$ signal loss below $1 \mathrm{kHz}$. This length is less than the difference between the two adjacent prosthesis sizes which measures about 250 $\mu \mathrm{m}$ (standard titanium prostheses). Clinical studies have also shown this typical air bone gap at low frequencies (39). Any pretension in the reconstruction with the rigid TORP was tried to be as good as this could be visually controlled. For this purpose, the connection point of the prosthesis head at the malleus handle was varied to achieve a good trade-off between position and pretension. The flexible TORP allowed small variations in length by deflecting the prosthesis. This way the prosthesis could always be placed with only little pretension. The investigator, an experienced otosurgeon, judged that the placement of the flexible TORP was slightly easier than that of the rigid TORP. The use of a cartilage shoe, an omega connector or an osseointegrated footplate anchor is recommended for better stability $(40 ; 32 ; 41)$. These provide a second joint in the reconstructed chain, preventing the lateral displacement of the prosthesis at the footplate and maintain the connection point at the footplate during pressure changes in the tympanic cavity.

Directly after implantation, the mean METF of both reconstructions (flexible and rigid TORP) were close to the intact middle ear (see Fig. 4 and Table 1). This was consistent with a study involving a previous version of the flexible TORP (11). The worst reconstruction with a rigid TORP, however, had about 10 $\mathrm{dB}$ more reduction in the METF than the worst reconstruction with a flexible TORP in a frequency range below $1 \mathrm{kHz}$ (Fig. 4). The reconstructions with the flexible TORP never showed an METF reduction of more than $8 \mathrm{~dB}$. This is due to the much better compliance of the new TORP design (27). It ensures that even with much longer prostheses, the pretension in the annular ligament is kept low (6). 
Other prostheses developments provide manual adjustment of the length during surgery $(16 ; 18)$. These allow for length adjustment freely with more degrees of freedom than with the predefined standard length prostheses. Nevertheless, such a procedure of length adjustment is still dependent on the surgeon's experience, because it is currently not possible to measure the real stiffness or the best fitting length during surgery. Nonetheless, individual length adjustment can be beneficial, but a standardized auto-adjusting prosthesis reduces the influence of the surgeon and should be preferred for assuring reproducibility and error minimization.

Yamada and Goode (17) introduced a prosthesis with an added piece of sponge. The viscoelastic material behavior of the sponge did not alter the METF compared to a standard prosthesis and it allowed a $0.45 \mathrm{~mm}$ increase in prosthesis length with less than $10 \mathrm{~dB}$ of METF reduction at frequencies below $1 \mathrm{kHz}$. These results are very similar to the results obtained with our new flexible TORP. The sponge used in that study was not yet biocompatible. A biocompatible sponge with the same viscoelastic material behavior could be an alternative to the flexible joint.

\subsection{Stability of the reconstruction}

A reconstruction of the ossicular chain must not only restore the normal METF, but ensure the reconstruction's long term stability as well. The main risks for prostheses dislocation are temporary or permanent static pressure changes (Valsalva maneuver, flights and tube ventilation disorders). Ruhl et al. (42) attributed a negative surgical outcome partly to positive pressure occurrences in the TC. The new flexible TORP could compensate movements of the tympanic membrane at about $+/-2.5 \mathrm{kPa}$. After three such load cycles, the METFs with the flexible TORP showed nearly no difference compared to the METFs directly after implantation (see Fig. 5 and Table 1). The rigid TORP seemed to lower the pressure load by repositioning, leading to decreased METF magnitudes. In general, this may have also caused larger METF standard deviations of the rigid TORP in comparison to the flexible TORP. As both TORP types (flexible and rigid) had the same plate/foot and were connected the same way to the $\mathrm{TM} /$ malleus handle as well as the stapes footplate, the better stability of the flexible TORP could only be attributed to the flexible joint in the prosthesis shaft. The deflection of the flexible TORP reduces the force at the connection points of the prosthesis and the ossicular chain since the TM introduces more load.

\subsection{METF at ambient pressure differences}

In case of ventilation tube disorders, the middle ear may be exposed to permanent under- pressure. With the application of ambient pressure, the METF of the intact middle revealed the characteristic reduction at low frequencies (up to $1 \mathrm{kHz}$ ) and a slight increase at frequencies above $2 \mathrm{kHz}$, as reported in literature (43). The METF of the reconstruction with the flexible TORP at negative pressure inside the TC was nearly equal to the METF of the intact ME. On the contrary, the METF with the rigid TORP showed considerably larger reductions (see Fig. 6, Fig. 7 and Table 1). This indicated that the flexible joint in the prosthesis mimicked the function of the ossicular joints. It reduced the load at the stapes annular ligament and thus caused less stiffening of this ligament.

At positive pressure in the TC, both prosthesis types became temporarily disconnected in some cases as the METF reduced by $30-40 \mathrm{~dB}$, which could be attributed to an interruption of the ossicular chain (44). Even the length variability of the flexible TORP could not compensate for the large tympanic membrane movements. The ambient pressure differences in this study were limited to $2.5 \mathrm{kPa}$, but they may be much higher in normal life. So far, there are no data on the transfer characteristics of the reconstructed middle ear at ambient pressure changes above $2.5 \mathrm{kPa}$. The results of this study also cannot be extrapolated to higher pressure due to nonlinear middle ear mechanics at high ambient pressure.

\section{Conclusions}

The METFs of the reconstructed ears by using the flexible TORP were equal or better than the METFs for the standard rigid TORP at normal pressure. In ears with ventilation disorders (negative pressure in the tympanic cavity), the flexible TORP mimicked the functionality of the intact chain. Then, an ossicular chain reconstruction with the flexible TORP tended to be more stable (in terms of prosthesis 
position and coupling) than a reconstruction with the rigid TORP. The findings may be considered preliminary as this experimental study was limited to just one of the many different possible situations of type III tympanoplasty and it involved a small sample size. Nevertheless, the results with the flexible TORP were promising and could encourage further investigations on such prostheses.

\section{References}

1. Geyer G, Rocker J. Results after rebuilding the ossicular chain using the autogenous incus, ionomer cement and titanium implants (tympanoplasty type iii). Laryngo Rhino Otol 2002; 81(3): 164-170

2. Beutner D, Hüttenbrink KB. Passive and active middle ear implants. Laryngo Rhino Otol 2009; 88 Suppl 1: 32-47

3. Zahnert T. Reconstruction of the middle ear with passive implants. HNO 2011; 59(10): 964-973

4. Schmerber S, Troussier J, Dumas G, Lavieille JP, Qui Nguyen D. Hearing results with the titanium ossicular replacement prostheses. Eur Arch Otorhinolaryngol 2006; 263(4): 347-354

5. Turck C, Brandes G, Krueger I, et al. Histological evaluation of novel ossicular chain replacement prostheses: an animal study in rabbits. Acta Otolaryngol 2007; 127(8): 801-808

6. Neudert M, Bornitz M, Lasurashvili N, Schmidt U, Beleites T, Zahnert T. Impact of Prosthesis Length on Tympanic Membrane's and Annular Ligament's Stiffness and the Resulting Middle Ear Sound Transmission. Otol Neurotol 2016; 37: e369-e376

7. Padurariu S, De Greef D, Jacobsen H, Kamavuako EN, Dirckx JJJ, Gaihede M. Pressure buffering by the tympanic membrane. In vivo measurements of middle ear pressure fluctuations during elevator motion. Hear Res 2015; 340: 113-120

8. Wehrs RE. Aeration of the middle ear and mastoid in tympanoplasty. Laryngoscope 1981; 91: 14631468

9. Pau HW. Inner Ear Damage in Torp-Operated Ears: Experimental Study on Danger from Environmental Air Pressure Changes. Ann Otol Rhinol Laryngol 1999; 108: 745-749

10. Yung M. Long-Term Results of Ossiculoplasty: Reasons for Surgical Failure. Otol Neurotol 2006; 27: 20-26

11. Arechvo I, Bornitz M, Lasurashvili N, Zahnert T, Beleites T. New total ossicular replacement prostheses with a resilient joint: experimental data from human temporal bones. Otol Neurotol 2012; 33: $60-66$

12. Yung M, Vowler SL. Long-Term Results in Ossiculoplasty: An Analysis of Prognostic Factors. Otol Neurotol 2006; 27: 874-881

13. Lüers JC, Beutner D, Hüttenbrink KB. Reconstruction of the ossicular chain-current strategies. Laryngorhinootologie 2010, 89, 172-81; quiz 182-3

14. Lailach S, Zahnert T, Lasurashvili N, Kemper M, Beleites T, Neudert M. Hearing outcome after sequential cholesteatoma surgery. Eur Arch Otorhinolaryngol 2015; 273: 2035-2046

15. Ihrle S, Gerig R, Dobrev I, et al. Biomechanics of the Incudo-Malleolar-Joint -Experimental Investigations for Quasi-Static Loads. Hear Res 2015; 340: 69-78

16. Zhao S, Hato N, Goode RL. Experimental study of an adjustable-length titanium ossicular prosthesis in a temporal bone model. Acta Oto-Laryngologica 2005; 125: 33-37

17. Yamada H, Goode R. A Self-Adjusting Ossicular Prosthesis Containing Polyurethane Sponge. Otol Neurotol 2010; 31: 1404-1408

18. Gottlieb PK, Li X, Monfared A, Blevins N, Puria S. First results of a novel adjustable-length ossicular reconstruction prosthesis in temporal bones. Laryngoscope 2016; 126: 2559-2564

19. Beleites T, Bornitz $M$, Offergeld C, Neudert M, Hüttenbrink KB, Zahnert T. Experimental investigations on middle ear prostheses with an integrated micro joint. Laryngo-Rhino-Otol 2007; 86: 649-654

20. Arechvo I, Zahnert T, Bornitz M, et al. The ostrich middle ear for developing an ideal ossicular replacement prosthesis. Eur Arch Otorhinolaryngol 2012a; 270: 37-44

21. Gostian AO, Pazen D, Lüers JC, Huttenbrink KB, Beutner D. Titanium ball joint total ossicular replacement prosthesis-experimental evaluation and midterm clinical results. Hear Res 2013; 301: 100-104 
22. Ringeval S, Fortunier R, Forest B, Martin C. Influence of the shape and material on the behaviour of a total ossicular replacement prosthesis. Acta Oto-Laryngologica 2004; 124: 789-792

23. Yung M. Titanium prosthesis with malleus notch: a study of its 'user-friendliness'. J Laryngol Otol 2007; 121: 938-942

24. Beutner D, Lüers JC, Bornitz M, Zahnert T, Hüttenbrink KB. Titanium Clip Ball Joint: A Partial Ossicular Reconstruction Prosthesis. Otol Neurotol 2011; 32: 646-649

25. Zahnert T. Laser in der Ohrforschung. Laryngo Rhino Otol 2003; 82 (1): 157-180

26. Bornitz $M$, Zahnert T, Hüttenbrink KB. Design considerations for length variable prostheses - Finite Element model simulations, Proceedings of the 3rd International Symposium Middle Ear Mechanics in Research and Otology (MEMRO), July 9-12, Matsuyama, Ehime, Japan, 2003, World Scientific Publishing, 2004; 153-160

27. Stoppe T, Bornitz M, Lasurashvili N, Sauer K, Zahnert T, Beleites T. Middle ear reconstruction with a flexible prosthesis. Curr Dir Biomed Eng 2017; 3 (2), 143-146

28. Lauxmann M. Nichtlineare Modellierung des Mittelohrs und seiner angrenzenden Strukturen. SHAKER Verlag, 2012; Band 27

29. Lauxmann M, Eiber A, Haag F, Ihrle S. Nonlinear stiffness characteristics of the annular ligament. J Acoust Soc Am 2014; 136: 1756

30. Koch M, Essinger TM, Stoppe T, Lasurashvili N, Bornitz M, Zahnert T. Fully implantable hearing aid in the incudostapedial joint gap. Hear Res 2016; 340: 169-178

31. Neudert M, Berner M, Bornitz M, Beleites T, Ney M, Zahnert, T. Osseointegration of prostheses on the stapes footplate: evaluation of the biomechanical feasibility by using a finite element model. $J$ Assoc Res Otolaryngol 2007; 8, 411-421

32. Schmid G, Steinhardt U, Heckmann W. The omega connector-a module for jointed coupling of titanium total prostheses in the middle ear. Laryngorhinootologie 2009; 88(12): 782-788

33. Mantei T, Chatzimichalis M, Sim JH, Schrepfer T, Vorburger M, Huber AM. Ossiculoplasty with total ossicular replacement prosthesis and Omega Connector: early clinical results and functional measurements. Otol Neurotol 2011; 32: 1102-1107

34. Rosowski JJ, Chien W, Ravicz ME, Merchant SN. Testing a method for quantifying the output of implantable middle ear hearing devices. Audiol Neurootol 2007; 12: 265-276

35. Hüttenbrink KB. Zur Rekonstruktion des Schallleitungsapparates unter biomechanischen Gesichtspunkten. Laryngo-Rhino-Otol 2000; 79: 23-51

36. Gea SLR. The application of microtomography in research of middle ear mechanics of gerbil and human at static pressure changes. Ph.D. thesis 2010, University of Antwerp

37. Committee on Hearing and Equilibrium. Guidelines for the evaluation of results of treatment of conductive hearing loss. Otolaryngol Head Neck Surg. 1995;113(3):186-187

38. Bance M, Morris DP, Vanwijhe RG, Kiefte M, Funnell WRJ. Comparison of the mechanical performance of ossiculoplasty using a prosthetic malleus-to-stapes head with a tympanic membraneto-stapes head assembly in a human cadaveric middle ear model. Otol Neurotol 2004; 25: 903-909

39. Truy E, Naiman A, Pavillon C, Abedipour D, Lina-Granade G, Rabilloud M. Hydroxyapatite Versus Titanium Ossiculoplasty. Otol Neurotol 2007; 28: 492-498

40. Beutner D, Lüers JC, Huttenbrink KB. Cartilage 'shoe': a new technique for stabilisation of titanium total ossicular replacement prosthesis at centre of stapes footplate. J Laryngol Otol 2008; 122: 682686

41. Neudert M, Beleites T, Ney M, et al. Osseointegration of Titanium Prostheses on the Stapes Footplate. J Assoc Res Otolaryngol 2010; 11: 161-171

42. Ruhl DS, Tolisano AM, Kesser BW, Hashisaki GT, Camacho M. Use of Positive Airway Pressure Following Middle Ear Surgery: A Practice Survey of Otologists. Otol Neurotol 2017; 38: 134-137

43. Murakami S, Gyo K, Goode RL. Effect of middle ear pressure change on middle ear mechanics. Acta Otolaryngol 1997; 117: 390-395

44. Rosowski JJ, Mehta RP, Merchant SN. Diagnostic utility of laser-Doppler vibrometry in conductive hearing loss with normal tympanic membrane. Otol Neurotol 2003; 24: 165-175 